\title{
Using lunar observations to validate pointing accuracy and geolocation, detector sensitivity stability and static point response of the CERES instruments
}

\author{
Janet Daniels $^{1}$, G. Louis Smith ${ }^{1}$, Kory J. Priestley ${ }^{2}$ and Susan Thomas ${ }^{1}$ \\ 1. Science Systems and Applications, Inc., 1 Enterprise Pkwy, Hampton, VA \\ 2. Langley Research Center, NASA, Mail Stop 420, Hampton, VA 23681, USA
}

\begin{abstract}
Validation of in-orbit instrument performance is a function of stability in both instrument and calibration source. This paper describes a method using lunar observations scanning near full moon by the Clouds and Earth Radiant Energy System (CERES) instruments. The Moon offers an external source whose signal variance is predictable and non-degrading. From 2006 to present, these in-orbit observations have become standardized and compiled for the Flight Models -1 and -2 aboard the Terra satellite, for Flight Models-3 and -4 aboard the Aqua satellite, and beginning 2012, for Flight Model-5 aboard Suomi-NPP. Instrument performance measurements studied are detector sensitivity stability, pointing accuracy and static detector point response function. This validation method also shows trends per CERES data channel of $0.8 \%$ per decade or less for Flight Models 1-4. Using instrument gimbal data and computed lunar position, the pointing error of each detector telescope, the accuracy and consistency of the alignment between the detectors can be determined. The maximum pointing error was $0.2^{\circ}$ in azimuth and $0.17^{\circ}$ in elevation which corresponds to an error in geolocation near nadir of $2.09 \mathrm{~km}$. With the exception of one detector, all instruments were found to have consistent detector alignment from 2006 to present. All alignment error was within $0.1^{\circ}$ with most detector telescopes showing a consistent alignment offset of less than $0.02^{\circ}$.
\end{abstract}

Keywords: Aqua, calibration, CERES, Earth Radiation Budget, EOS, Moon, radiometry, remote sensing, Terra, validation

\section{INTRODUCTION}

Five CERES Flight Model (FM) instruments are currently in-orbit and operational with FMs-1 and -2 on Earth Observation System (EOS) AM-1 satellite, Terra; FMs-3 and -4 on Earth Observation System (EOS) PM-1 satellite, Aqua; and FM-5 on Suomi National Polar-orbiting Platform (NPP). The CERES instruments are three-channel radiometers that measure solar radiation reflected by the Earth, radiation emitted by the Earth, and radiation in the $\mathrm{CO}_{2}$ window band of the atmosphere. The largest uncertainty in understanding climate sensitivity is the effect of cloud feedback, and the CERES instruments assist in constraining this uncertainty by providing measurements of cloud radiative forcing ${ }^{1}$. Studies of the Radiation Budget Climate Data Record (RBCDR) have shown that permitted error ranges allowed in the radiation fluxes over the Earth should be within $1 \%$ for shortwave fluxes and $0.5 \%$ for outgoing longwave fluxes ${ }^{2}$. This level of accuracy requires that CERES instruments be calibrated frequently in orbit, and these results must be validated by inter-instrument comparisons to provide confidence for the continuity of the $\mathrm{RBCDR}^{3}$.

One of the challenges to any long-term calibration study is separating changes in the calibration source from changes in the instrument. Unlike internal calibration sources, the Moon is a non-degrading external source whose signal variance is predictable. This paper begins with a brief overview of the CERES instrument and a more detailed description of its telescopes and detectors. Next, lunar observation method is described. The sections afterward discuss the use of lunar

Corresponding Author: Janet Daniels, NASA Langley Research Center, E-mail: Janet.1.Daniels@ nasa.gov Phone: Office: (757) 864-2778, Mobile: (757) 345-9513 
observations in determining changes in CERES instrument pointing accuracy and detector alignment, detector stability, point response function, and potential trends in CERES radiances.

\section{THE CERES INSTRUMENT}

CERES is a bi-axial scanning instrument with three thermistor-bolometer channels. A description of the CERES radiometer is found in Wielicki et al. (1996) and in Figure 1. The shortwave channel measures solar radiation reflected by the Earth from 0.3 to $<0.5$ microns; the total channel measures radiation emitted by the Earth from 0.3 to $>100$ microns; and the window channel measures radiation in the $\mathrm{CO}_{2}$ window range of 8 to 12 microns. Each bolometer is located behind the focal plane of a Cassegrain telescope in which an elongated hexagonal field stop has been inserted to constrain incident radiation into the field of view. All three channels rotate in elevation at a normal scan rate of $67.85 \mathrm{deg} / \mathrm{sec}$. The signal from each channel is sampled every $10 \mathrm{~ms}$ or every 0.6785 degrees.

Requirements for the instrument state that the three telescopes of each CERES instrument must be aligned so that all three of its detectors observe the same scene simultaneously. Scene classification for each CERES pixel is computed using imager data from instruments located on the same spacecraft as each CERES instrument. Moderate Resolution Imaging Spectro-radiometer (MODIS) data is combined with CERES FMs 1-4, and Visible Infrared Imaging Radiometer Suite (VIIRS) data is used for CERES FM-5. Imaging instrument pixels are an order of magnitude smaller than those of CERES, so it is necessary to know the response of the instrument to a point within the CERES field-of-view as it scans. This effect is denoted the point response function (PRF). The PRF is needed for CERES for two reasons: first, to locate the centroid of each measurement and to validate its position at the surface of the

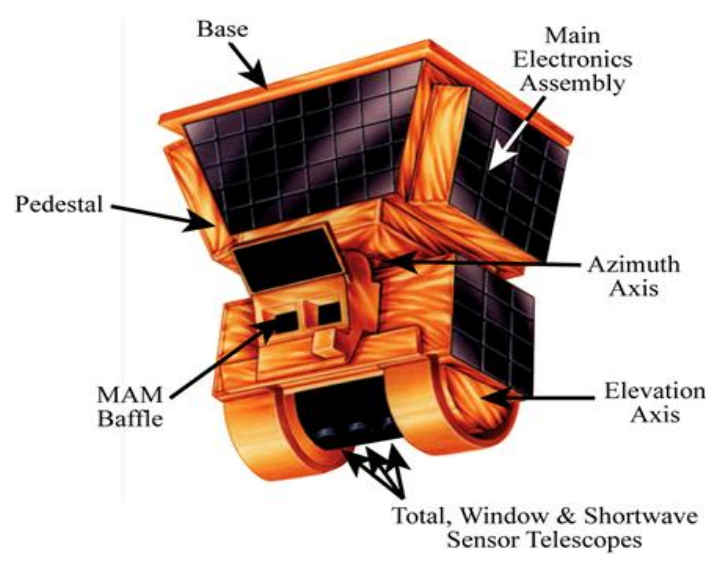

Figure 1. The CERES FM5 Instrument. Earth, and second, to use for applying higher resolution imager data with the CERES measurements.

During normal operations, radiation impinging on the detector causes a continuous increase in temperature through the detector. Figure 2 shows the various layers that make up each CERES detector. If all layers are perfectly bonded, the resulting detector gives uniform output across its entire surface, as seen in Figure $2 b$.
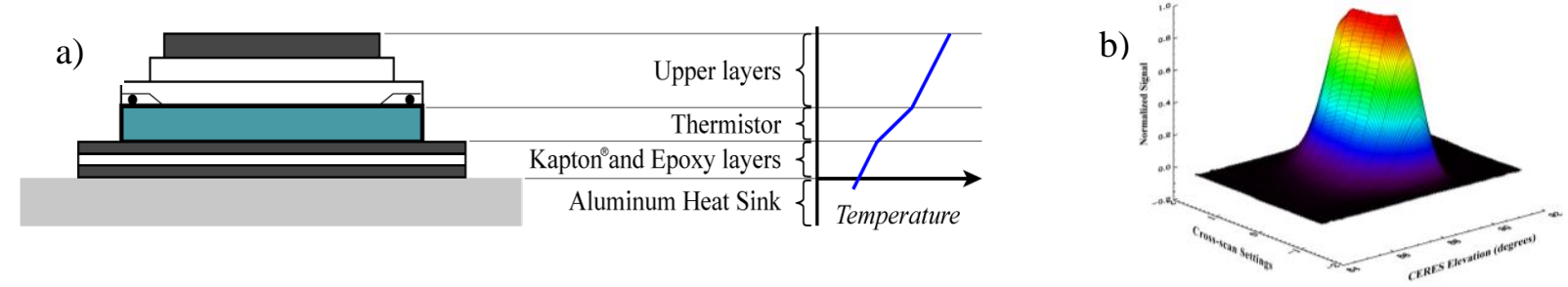

Figure 2. a) Basic cross-section of CERES detector layers, b) Output from CERES detector showing uniformity response.

If delamination occurs between the paint layer and the thermistor, the thermistor flake does not heat as efficiently, and the detector measures lower than it should. If delamination occurs between the semiconductor and the heat sink, the temperature increases because the conduction path has been broken causing a greater sensitivity at this location and the measurement registers higher values as shown in Figure 3.
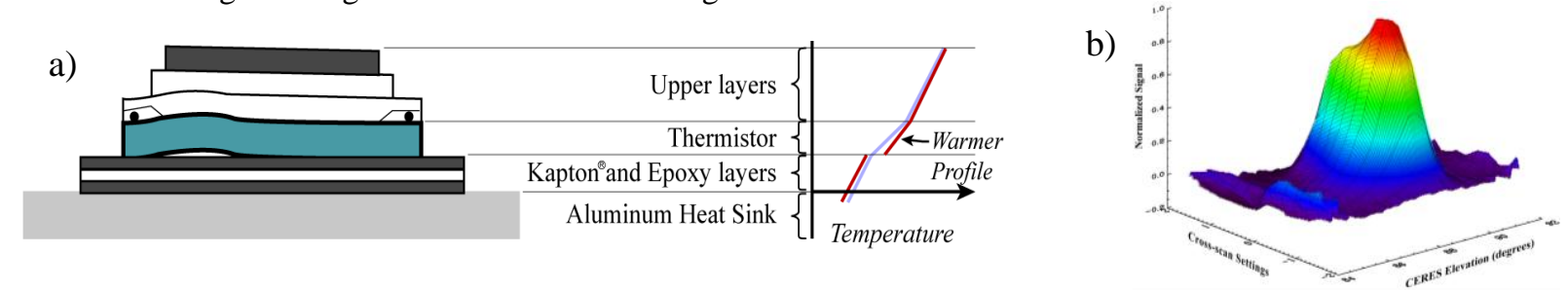

Figure 3. a) Cross-section of CERES detector layers showing delamination, b) Output from CERES detector showing resulting hot spot. 
Delamination was determined to have occurred between the thermistor and heat sink for the Window channel detector for FM2 and FM5. Offset from center, the PRF data appears as an increase or hot spot with respect to detector sensitivity. The FM4 shortwave channel malfunctioned in 2004 and, therefore, is not included in this study.

\section{LUNAR OBSERVATION METHOD}

Since 2006, lunar observations have been standardized for CERES instruments by scanning the moon during 5 orbit prior to full moon and 5 orbits after full moon. Each CERES instrument rotates in azimuth to bring the Moon into view between the nadir side of each instrument and the limb of the Earth. As each satellite moves along its orbit, the Moon sets below the limb of the Earth. CERES observes the Moon at $-17.78^{\circ}$ in elevation. A $12.9^{\circ}$ azimuth angle range is calculated for each orbit allowing the FOV to scan onto, across and off the lunar disk to obtain a spacelook which establishes a zero radiance reference for each individual scan at a rate of $4 \%$ until the Moon has passed from the plane of the FOV. An average of 10 scans is taken over a period of less than 1 minute in duration for each orbit. An illustration based on actual track data is shown in Figure 4. Satellite ephemeris and instrument position information are used to compute lunar position across the FOV. This method is discussed in detail in Daniels $(2014)^{10}$.

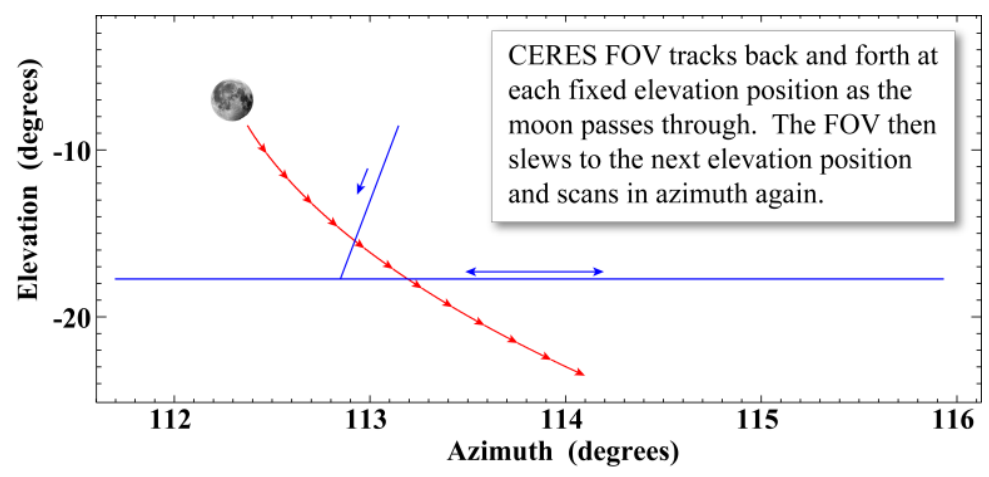

Figure 4. Apparent movement of Moon with respect to the CERES axis.

Figure 5 shows the moon within the field of view of CERES. The FOV is a hexagon $2.6^{\circ}$ across the corners and $1.3^{\circ}$ between the sides. From mean satellite-Moon distance, the Moon is a circle with diameter $0.52^{\circ}$. For in-orbit PRF calibrations, the Moon is used as the point source. As designed, the detector should have a uniform response to radiation over its surface, so that the static point response function is determined by the field stop. Azimuth scan-rate during lunar observations is faster than Moonset elevation sink-rate by an order of magnitude. These in-orbit observations are taken at a lower azimuth scanning rate when compared to ground testing, listed in Table 1. Therefore, data points are much closer than for standard pre-launch ground calibrations, and the detector can be mapped with greater precision.

Table 1. Ground vs. Lunar PRF settings

\begin{tabular}{|lcc|}
\hline & Ground & Lunar \\
\hline Point Source & $0.17^{\circ}$ & $0.52^{\circ}$ \\
\hline Scan Rate & $67.85^{\circ} / \mathrm{sec}$ & $4.0^{\circ} / \mathrm{sec}$ \\
\hline
\end{tabular}

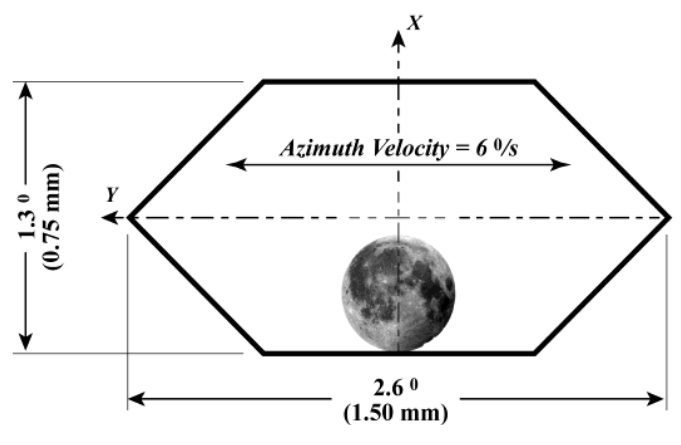

Figure 5. Moon in CERES field of view

Lunar phase angle is defined as the angle between the sub-solar point and the sub-satellite point on the Moon and is the dominant factor in lunar irradiance. These observations are limited in time to when the Moon is visible to the instrument at a specific instrument elevation angle. Because of this narrow time window per orbit, observing the moon at specific phase angles is not possible. Therefore, CERES lunar observations are timed to occur over lunar phase angle ranges of [-12 ${ }^{\circ}$ to $\left.-5^{\circ}\right]$ prior to fullest moon, fullest moon, and $\left[5^{\circ}\right.$ to $\left.12^{\circ}\right]$ after fullest moon. This series of 11 observations occur over a period of 18 hours centered at full moon and are each only minutes in duration. 
After lunar observations are complete, a number of orbital effects are removed from the data. Lunar spectral radiance changes due to variations in distance between Sun-to-Moon, lunar phase angle and spectral albedo over the surface. The amount of radiance as seen by the instrument detector is affected by the satellite-to-Moon distance. If the Moon were always oriented so that the instrument always saw the same view of the Moon, the spectral irradiance would vary only due to the position and distance to the Sun, the distance to the instrument and the solar phase angle. With these distances taken into account, the measurement would then vary due to changes of the gain of the channel or of the spectral response. However, the sub-satellite point (the point at which a line from instrument detector to the center of the Moon intersects the lunar surface) moves as the Moon moves around its elliptical orbit with nearly a constant rate of rotation. This change of orientation of the Moon as seen by the detector is called libration. The sub-solar point on the Moon also varies. The results of these effects are about $1 \%$ of the irradiance. Because of the variation in spectral albedo of the lunar surface, libration changes the spectral irradiance at the instrument.

The instrument response to radiation changes partly due to changes of the spectral responses of each channel ${ }^{5}$. Data from the first six months of operation are processed using ground calibrations. Internal calibration devices are used to calibrate the instruments about every three months thereafter. These data products are Edition $1 \mathrm{C}-\mathrm{V}$ (calibration and validation) and are used in this study. To get meaningful radiances from the Earth, it is necessary to account for the spectral responses of the channels, producing "unfiltered" radiances. This investigation examines the filtered radiances to validate the stability of the measurements but does not attempt to account for the spectral responses of the instruments to measure the Moon's irradiance.

\section{POINT RESPONSE FUNCTION}

The responses of the CERES detectors are not ideally uniform, but vary with location over each detector surface. The individual detector responses have been mapped using lunar observations by plotting the data in FOV elevation and azimuth to provide a detailed map of each detector ${ }^{6,7}$. To verify whether an adjustment in the point response function (PRF) is needed per detector, lunar calibrations were selected where multiple opposing scan slices occurred on each side of the detector. Values were adjusted when necessary to align the selected scans.

Due to the variation of detector data-coverage and signal intensity, the number of scans taken and the unpredictability of scan locations when more than one scan can be used for a given lunar calibration, verification of each adjustment was performed manually to ensure the correctness of the result. Figure 6 shows results from a correction in the FM2 Shortwave Channel PRF.
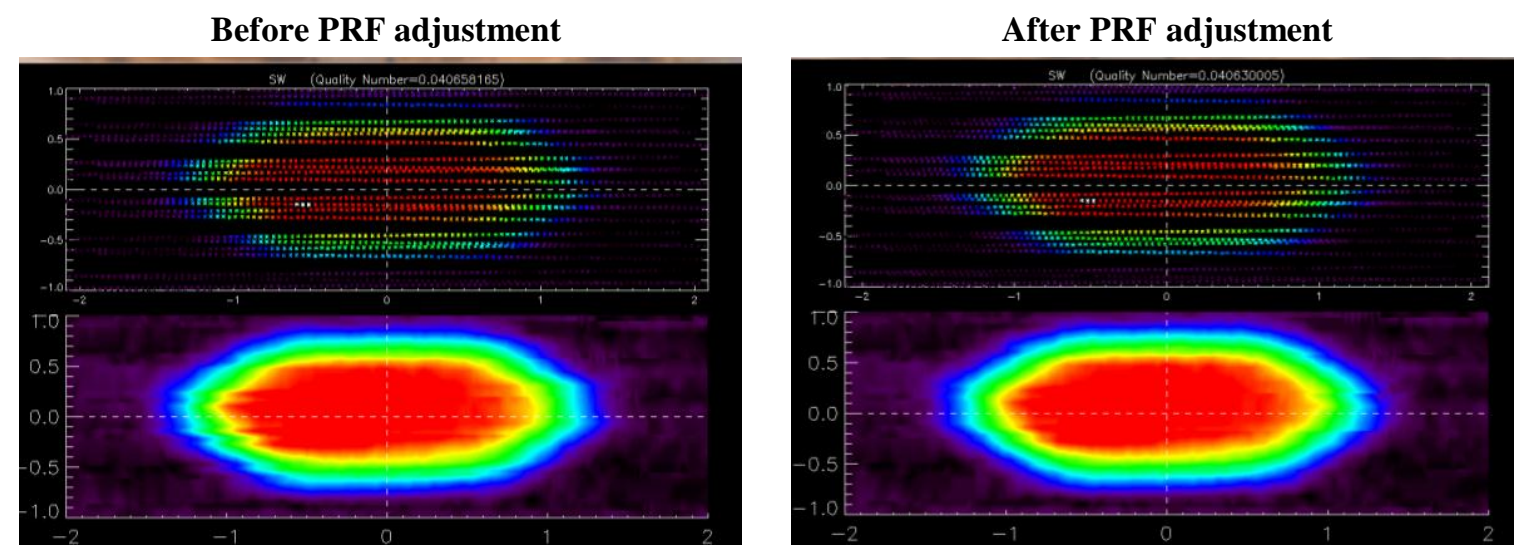

Figure 6. Detector output showing misalignment of signal prior to PRF adjustment (right), and after the correction is applied (left).

Lunar observations are used to verify that detector response remains constant over the mission by independently calculating the best PRF per orbit. Results listed in Table 2 show that CERES detectors on all satellite platforms have remained constant from 2006 to 2014 for each detector, although these in-orbit results are slightly different than those calculated during pre-launch ground calibrations. These small differences between ground and in-orbit PRF calibrations are likely due to differences in data sampling rate and not changes in detector response. Part of the window detector on FM2 is delaminated and thus exhibits a different point response from one side of the detector to the other. 
Table 2. Ground vs. Lunar PRF values (msec)

\begin{tabular}{|c|c|c|c|c|c|c|c|c|c|c|c|c|c|c|}
\hline \multirow{2}{*}{$\begin{array}{l}\text { Channel } \\
\text { PRF (msec) }\end{array}$} & \multicolumn{3}{|c|}{ FM1 } & \multicolumn{3}{|c|}{ FM2 } & \multicolumn{3}{|c|}{ FM3 } & \multicolumn{2}{|c|}{ FM4 } & \multicolumn{3}{|c|}{ FM5 } \\
\hline & TOT & SW & WN & TOT & SW & $\mathbf{W N}$ & TOT & SW & $\mathbf{W N}$ & TOT & WN & TOT & SW & $\mathbf{W N}$ \\
\hline Ground Cal & 21 & 22 & 22 & 24 & 22 & 22 & 22 & 25 & 23 & 23 & 25 & 23 & 25 & 24 \\
\hline 2006 Lunar Cal & 24 & 24 & 27 & 23 & 29 & $33 / 22$ & 29 & 28 & 28 & 30 & 28 & 25 & 25 & 25 \\
\hline 2014 Lunar Cal & 24 & 24 & 27 & 23 & 29 & $33 / 22$ & 29 & 28 & 28 & 30 & 28 & 25 & 25 & 25 \\
\hline$\Delta($ Ground -Lunar $)$ & -3 & -3 & -5 & 1 & -7 & $-11 / 0$ & -7 & -3 & -5 & -7 & -3 & -2 & 0 & -1 \\
\hline$\Delta$ Lunar (2006-2014) & 0 & 0 & 0 & 0 & 0 & 0 & 0 & 0 & 0 & 0 & 0 & 0 & 0 & 0 \\
\hline
\end{tabular}

Once the data is adjusted with respect to PRF, data from each orbit is translated into a standardized grid, FOV center for each detector can be calculated and detector alignment and pointing accuracy can be obtained. The range for this standard grid is $\left[-2^{\circ}, 2^{\circ}\right]$ in azimuth, and $\left[-1^{\circ}, 1^{\circ}\right]$ in elevation with $0.01^{\circ}$ spacing between points.

\section{DETECTOR ALIGNMENT AND POINTING ACCURACY}

Two methods used to calculate the FOV center location for each detector are Full-Width-Half-Maximum (FWHM) method and the Interpolation method ${ }^{8}$. FWHM method, described below, gives results on the physical center of the FOV regardless of variations in sensitivity across the surface of the detector. The Interpolation method shows the resulting signal center of the FOV.

\subsection{Full-Width-Half-Maximum (FWHM)}

As the CERES telescopes track across the Moon, a rapid change in signal occurs at the edge of the detector, and this signal change is used with the following method to detect the physical center of each detector. For each row and each column of gridded data, the maximum value per slice is found. This maximum value is divided in half, and the location on either side of maximum which best corresponds to this halved value is recorded. The mid-point between these two drop-off values is calculated to be the physical center of that slice across the detector. This midpoint array is calculated in azimuth and elevation separately. The final detector center is the mean of each array in azimuth and elevation and illustrated in Figure 7.

\subsection{Interpolation}

For a perfect detector, both the physical center and the signal center would be located in identical positions. However, detector sensitivity is not uniform, and the interpolation method is used to pinpoint how this non-uniformity of recorded incoming energy could affect location accuracy. The mid-point of the interpolated detector signal across each slice in azimuth and elevation is calculated. The final signal center is the mean of

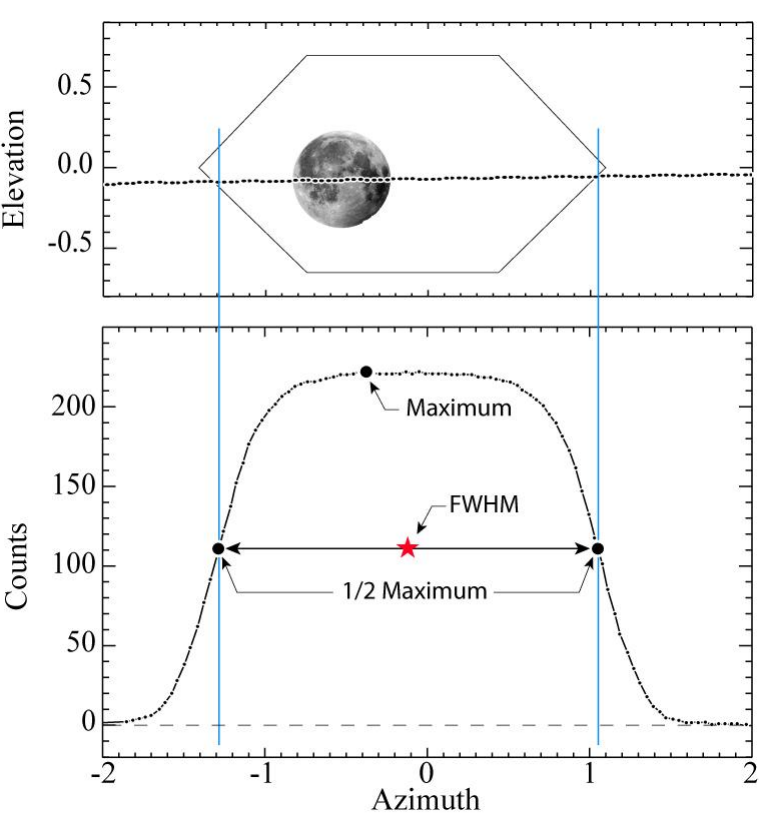

Figure 7. Physical center of detector is computed using Full-Width-Half-Maximum method each array bounded by in azimuth and in elevation.

\subsection{Physical Detector Center vs. Detector Signal Center}

Validation plots are created for each orbit and reviewed. Figure 8 is a sample from FM2 for one orbit of lunar calibration data from 2004. From left to right, Total, Shortwave and Window channel detectors are shown mapped in azimuth and elevation. White lines show results of calculated azimuth and elevation center positions using the FWHM method for physical detector center. The blue lines show results using the interpolation method for detector signal center. Total and 
shortwave detectors show examples of the two centers located in the same place, while the known delamination of the window detector illustrates an extreme example of the two centers in different locations. The green diamond marks signal-center, and the black square marks physical-center in each detector.

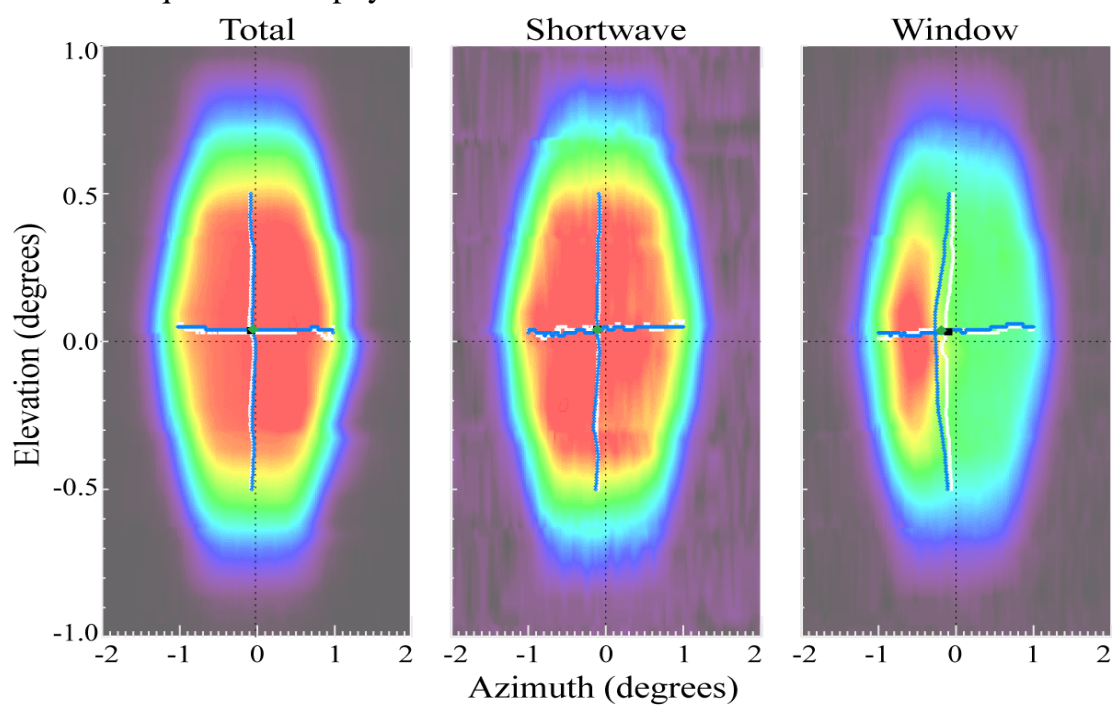

Figure 8. FM2 Total, shortwave and window channel detectors showing data from one orbit of lunar observations. The total detector shows near-perfect results where the physical center (calculated by FWHM) overlays the signal center as calculated by interpolation method. The window detector with its known delamination illustrates where physical center and signal center do not agree.

\subsection{Results for Pointing Accuracy and Detector Alignment}

Changes in pointing accuracy are studied by comparing FWHM center values of shortwave and window detectors with that of the total detector. Telescope alignment shows good consistency for all instruments with the exception of a small noticeable trend in FM3 window detector, included in Figure 9 with results from FM1 for comparison.

To retrieve the required radiances, the CERES instruments require that all three channel detectors observe the same location simultaneously. Using results from FWHM, the alignments of the shortwave and window channels are compared with that of the total channel with Table 3 containing the resulting alignment errors found.
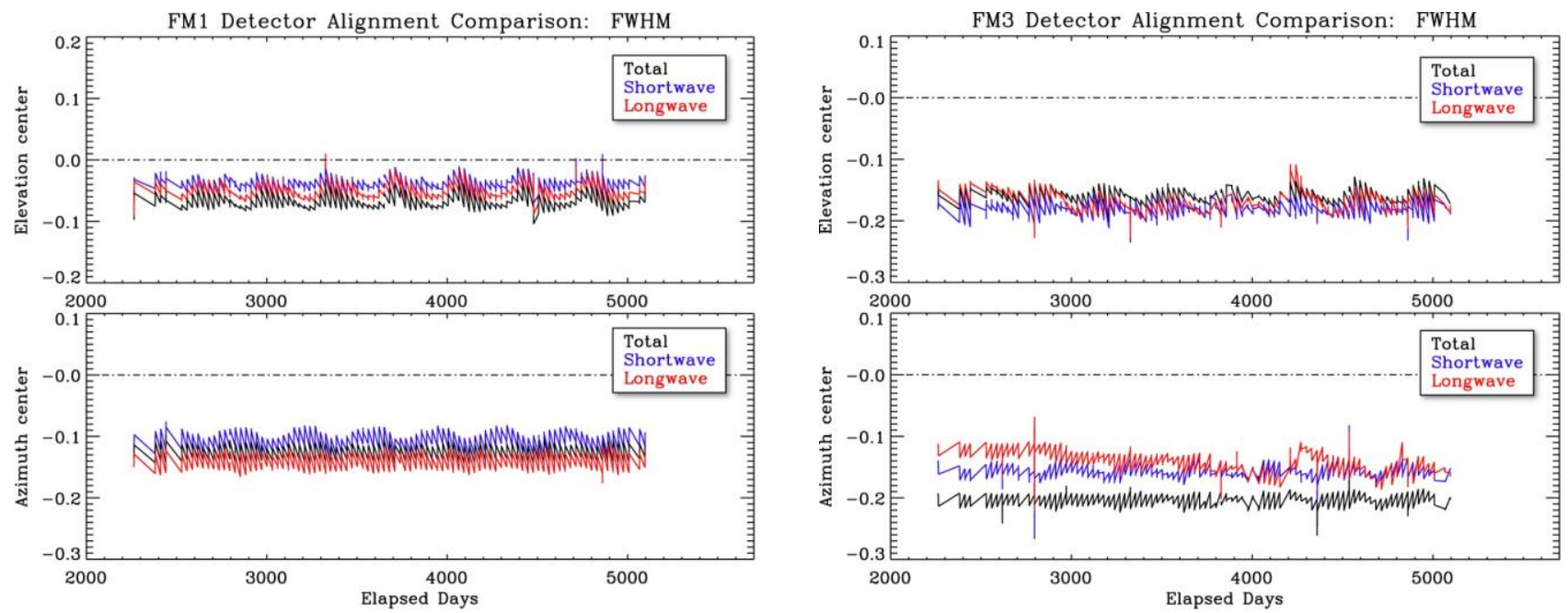

Figure 9. FM1 center detector data are shown on the left, and FM3 results are shown in the right. Elevation and azimuth data are shown in degrees. FM3 exhibits perturbations in azimuth alignment and lesser variations in elevation for the window detector. 
Table 3: Alignment error calculated using lunar observations.

\begin{tabular}{|c|c|r|r|rr|}
\hline \multirow{2}{*}{ Flight Model } & \multirow{2}{*}{ Channel } & \multicolumn{2}{|c|}{$\begin{array}{c}\text { AZ Alignment Error } \\
\text { (degrees) }\end{array}$} & \multicolumn{2}{c|}{$\begin{array}{c}\text { EL Alignment Error } \\
\text { (degrees) }\end{array}$} \\
\cline { 3 - 6 } & & FWHM & Interp & FWHM & Interp \\
\hline $\mathbf{1}$ & SW & -0.019 & -0.020 & -0.028 & -0.024 \\
\cline { 2 - 6 } 996 orbits & WN & 0.015 & 0.036 & -0.017 & -0.023 \\
\hline $\begin{array}{c}\mathbf{2} \\
1004 \text { orbits }\end{array}$ & SW & 0.052 & 0.082 & 0.001 & 0.005 \\
\cline { 2 - 6 } $\mathbf{3}$ & WN & 0.040 & 0.159 & 0.001 & 0.003 \\
\hline 810 orbits & WN & -0.046 & -0.056 & 0.019 & 0.016 \\
\hline $\begin{array}{c}\mathbf{4} \\
785 \text { orbits }\end{array}$ & WN & -0.064 & -0.092 & 0.009 & 0.017 \\
\hline $\mathbf{5}$ & SW & 0.008 & -0.021 & 0.009 & -0.026 \\
\cline { 2 - 6 } 216 orbits & WN & -0.024 & -0.060 & 0.008 & -0.009 \\
\hline
\end{tabular}

Results show that alignment errors are within $0.07^{\circ}$ or less. Since the radius of the blur circle produced by spherical mirrors of the telescope is $0.16^{\circ}$, these alignment errors are negligible for all five instruments ${ }^{9}$. Center detector locations for both methods with standard deviation calculated for FWHM are listed in Table 4 for CERES Flight Models 1 through 5. A measurement of detector stability can be calculated by comparing both locations with respect to each other. If there are changes across the surface of the detector with time, a trend could occur in detector signal center. The difference between the physical center and the signal center was trended and the resulting near-zero values of the slope change per decade are listed in Table 4, as well.

Table 4: Pointing accuracy determined by FWHM and interpolation methods using lunar observations.

\begin{tabular}{|c|c|c|c|c|c|c|c|c|c|}
\hline \multirow{2}{*}{$\begin{array}{l}\text { Flight } \\
\text { Model }\end{array}$} & \multirow[t]{2}{*}{ Channel } & \multicolumn{3}{|c|}{$\begin{array}{c}\text { Pointing Accuracy AZ } \\
\text { (degrees) }\end{array}$} & \multirow{2}{*}{$\begin{array}{c}\text { Slope } \\
\Delta\end{array}$} & \multicolumn{3}{|c|}{$\begin{array}{c}\text { Pointing Accuracy EL } \\
\text { (degrees) }\end{array}$} & \multirow{2}{*}{$\begin{array}{l}\text { Slope } \\
\Delta\end{array}$} \\
\hline & & FWHM & Interp & StDev & & FWHM & Interp & StDev & \\
\hline \multirow{3}{*}{$\begin{array}{c}1 \\
996 \text { orbits }\end{array}$} & TOT & -0.128 & -0.120 & 0.011 & $3 e-9$ & -0.064 & -0.060 & 0.011 & $1 \mathrm{e}-7$ \\
\hline & SW & -0.108 & -0.101 & 0.012 & $2 e-7$ & -0.040 & -0.036 & 0.011 & $-3 e-7$ \\
\hline & $\mathrm{WN}$ & -0.142 & -0.157 & 0.012 & $7 e-8$ & -0.051 & -0.037 & 0.012 & $-4 \mathrm{e}-7$ \\
\hline \multirow{3}{*}{$\begin{array}{c}2 \\
1004 \text { orbits }\end{array}$} & TOT & -0.067 & -0.050 & 0.011 & $6 e-8$ & 0.020 & 0.028 & 0.017 & $1 \mathrm{e}-7$ \\
\hline & SW & -0.118 & -0.118 & 0.013 & $-4 e-8$ & 0.019 & 0.020 & 0.018 & $5 e-7$ \\
\hline & WN & -0.106 & -0.199 & 0.012 & $-2 e-7$ & 0.018 & 0.025 & 0.021 & $1 \mathrm{e}-7$ \\
\hline \multirow{3}{*}{$\begin{array}{c}3 \\
810 \text { orbits }\end{array}$} & TOT & -0.201 & -0.210 & 0.009 & $3 e-8$ & -0.161 & -0.148 & 0.011 & $-2 \mathrm{e}-7$ \\
\hline & SW & -0.155 & -0.153 & 0.010 & $2 e-7$ & -0.180 & -0.162 & 0.012 & $-4 e-8$ \\
\hline & $\mathrm{WN}$ & -0.137 & -0.117 & 0.017 & $2 e-7$ & -0.170 & -0.161 & 0.017 & $-5 e-7$ \\
\hline \multirow{2}{*}{$\begin{array}{c}\mathbf{4} \\
785 \text { orbits }\end{array}$} & TOT & -0.145 & -0.134 & 0.010 & $2 e-7$ & 0.093 & 0.089 & 0.011 & $-1 e-7$ \\
\hline & $\mathrm{WN}$ & -0.153 & -0.125 & 0.011 & $3 e-7$ & 0.114 & 0.116 & 0.012 & $-1 e-7$ \\
\hline \multirow{3}{*}{$\begin{array}{c}\mathbf{5} \\
216 \text { orbits }\end{array}$} & TOT & -0.142 & -0.113 & 0.007 & $-6 e-6$ & -0.122 & -0.116 & 0.062 & $4 \mathrm{e}-6$ \\
\hline & SW & -0.156 & -0.156 & 0.008 & $-6 e-7$ & -0.113 & -0.107 & 0.064 & $1 e-6$ \\
\hline & WN & -0.118 & -0.043 & 0.007 & $-5 e-6$ & -0.130 & -0.142 & 0.063 & $4 e-6$ \\
\hline
\end{tabular}

\subsection{Results for Geolocation Accuracy}

The effect of pointing errors can be used to calculate the error in geolocation of footprints. The CERES coordinate axes and rotations are shown in Figure 10. The z-axis corresponds to the azimuth axis of rotation, and the $\mathrm{x}$-axis is the axis of rotation in elevation. A rotation in elevation. A rotation in the $y$-axis corresponds to the tilt of the scan beam axis away 
from the horizontal plane. These calculations are described in detail in Daniels, et al. $(2014)^{8}$. Cross-track error can be calculated by

$$
\varepsilon_{C T}=s \alpha \sec \theta
$$

where $s$ is the slant range from the spacecraft to the scene, $\alpha$ is mean elevation error of the three instrument detectors, and $\theta$ is the angle from spacecraft to center of the Earth to the scene geolocation. At nadir, this equation can be simplified to

$$
\varepsilon_{C T}=h \alpha
$$

where $h$ is spacecraft altitude. Resulting geolocation errors at Nadir for CERES instruments 1 through 5 are listed in Table 5. Using Eq. (1), as the instruments scan to the limb as a function of viewing angle, cross-track errors are shown in Figure 11.

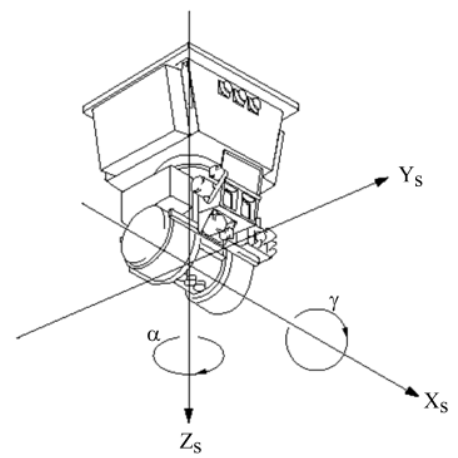

Figure 10. Instrument coordinate axes and rotations

Table 5: Geolocation calculations for lunar observations

\begin{tabular}{|c|c|cc|r|}
\hline \multirow{2}{*}{$\begin{array}{c}\text { Flight } \\
\text { Model }\end{array}$} & $\boldsymbol{h}$ & \multicolumn{2}{|c|}{$\begin{array}{c}\text { Alignment Error } \\
\text { (km) }\end{array}$} & \multicolumn{2}{|c|}{$\alpha$} & $\operatorname{StDegrees})$ & $\begin{array}{c}\varepsilon_{C T} \\
\text { Nadir } \\
(\mathbf{k m})\end{array}$ \\
\hline 1 & 705 & -0.052 & 0.011 & -0.64 \\
\hline 2 & 705 & 0.022 & 0.019 & 0.27 \\
\hline 3 & 705 & -0.170 & 0.013 & -2.09 \\
\hline 4 & 705 & 0.104 & 0.010 & 1.28 \\
\hline 5 & 824 & -0.122 & 0.007 & -1.75 \\
\hline
\end{tabular}

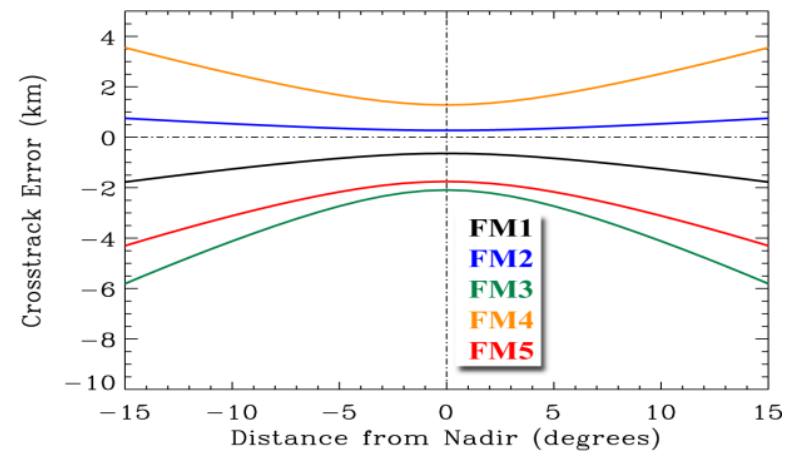

Figure 11. Cross-track error in geolocation due to distance of scene from nadir.

\section{VALIDATION OF INSTRUMENT CALIBRATIONS}

Validation of in-orbit instrument performance can also be done using lunar observations ${ }^{10}$. The Moon is an extremely stable, independent target which removes the question of calibration source uncertainty ${ }^{11}$. Data are adjusted for PRF and translated from an irregular to a standard angular grid for long-term trending and sensor output comparison. Orbital geometry effects are addressed next. Variations in spectral radiance of the lunar surface are cause by changes in the distance between Sun-to-Moon, phase angle over the lunar surface and spectral albedo. The amount of incoming energy to the detector is also a function of the distance from satellite-to-Moon. The last orbital effect addressed is the change in orientation of the Moon as seen by the detector which is called libration. Prior to correcting for these orbital effects, measurements of lunar irradiance by CERES vary by $20 \%$. The final trends per data channel show results where almost all of this variation has been removed. For this validation, a dataset spanning at least 2 years yields best results. Therefore, only data from FMs -1 through -4 are included in this section of the paper. 
Data per orbit per channel are numerically integrated into a single value for overall radiance. The resulting data array is normalized by the mean of the entire dataset. In order of magnitude, the orbital effects are found to be Satellite-Moon (SM) distance, Earth-Sun (ES) distance, lunar phase angle and lunar libration. SM and ES effects are adjusted using the inverse square. Figure 11 shows three plots of FM1 Total Channel data with the original integrated data, data corrected for SM, and data corrected for ES.

a)

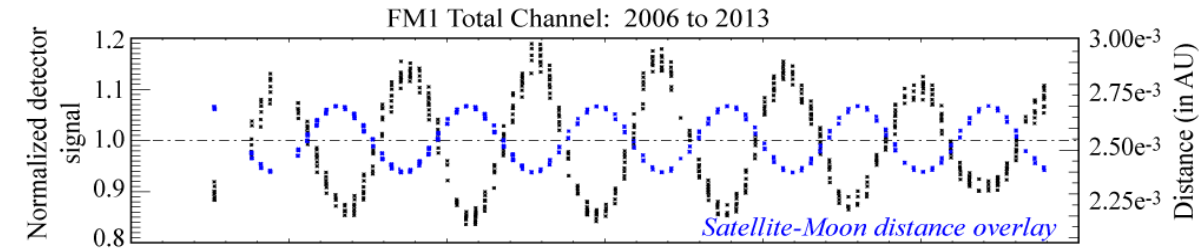

b)

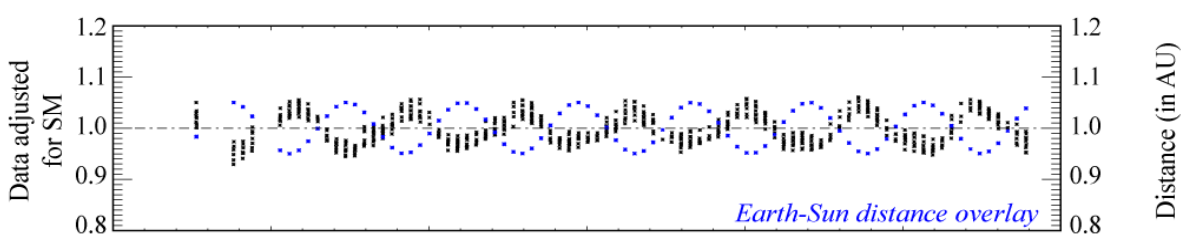

c)

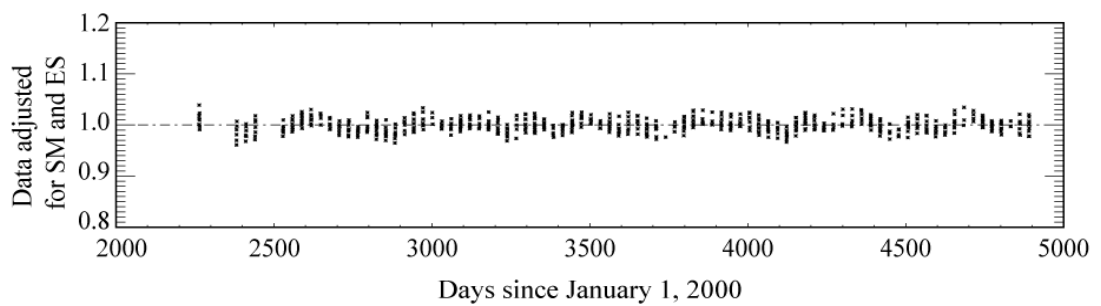

Figure 11. a) Variation of Total channel output (black, interpolated and normalized) and Satellite-Moon (SM) distance curve-shape overlaid (blue) with time, b) Variation of Satellite-Moon-adjusted FM-1 Total channel output (black) and Earth-Sun (ES) distance curve-shape overlaid (blue), c) FM-1 Total channel output after Earth-to Moon and Earth-to-Sun adjustment.

CERES instruments observe the Moon over phase angle ranges from $5^{\circ}$ to $12^{\circ}$. Over this range, the relation between detector response and phase angle is inversely proportional and presumed to be linear. Removal of the phase angle effect results in a decrease in detector variability per month and reveals a cyclic variation caused by libration of the Moon as seen by the instrument. Figure 12 illustrates the relation of phase angle to signal and the results of applying this adjustment.
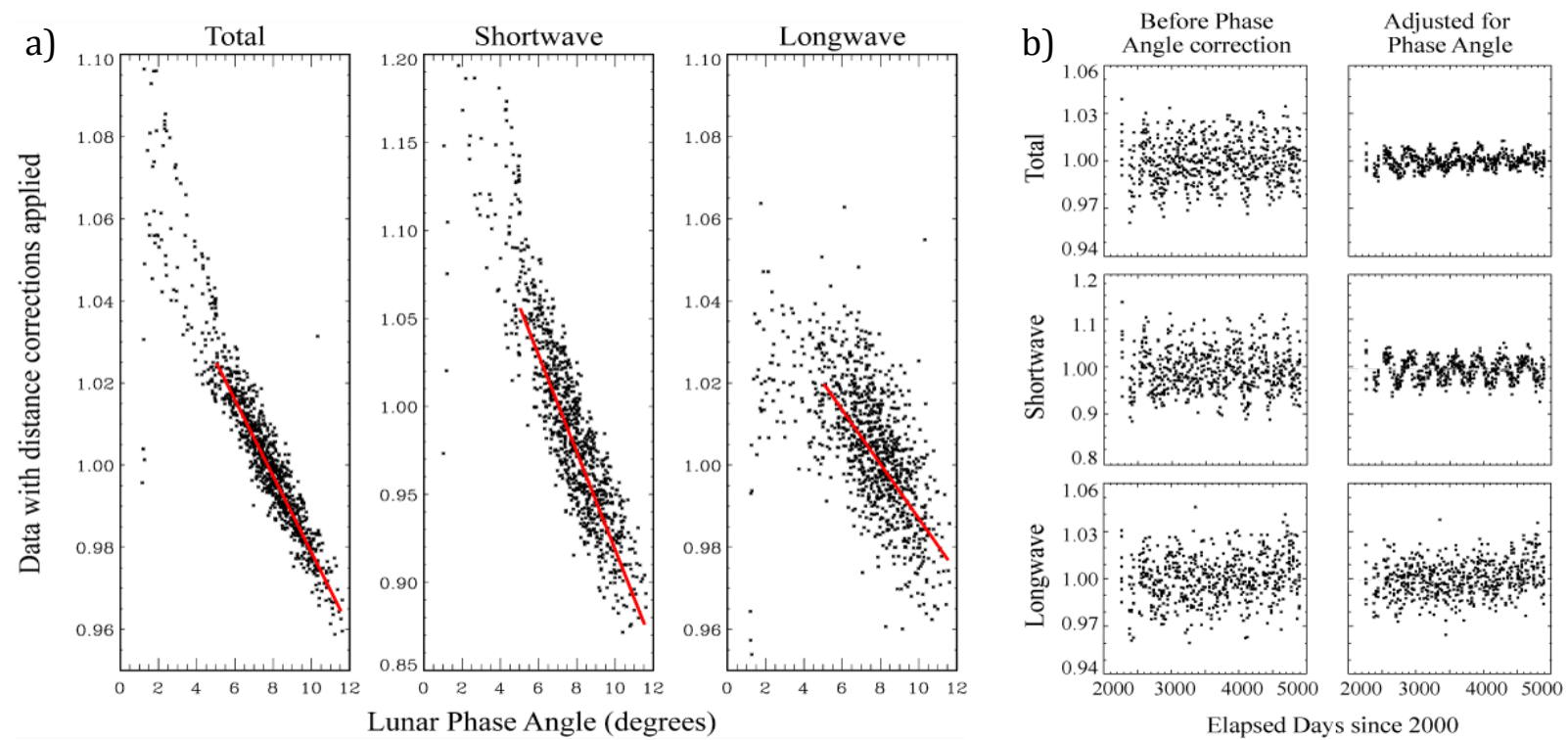

Figure 12. a) Lunar Phase Angle and CERES detector response per channel, b) CERES FM-1 detector responses before and after lunar phase angle adjustment 
The remaining cyclic variations of $\pm 1 \%$ for total channel and $\pm 5 \%$ for shortwave are an effect of libration as the proportion of areas of dark maria and bright terrae change in as viewed by the satellite. In Figure 13, detector data per orbit is shown in black and a $2^{\text {nd }}$ order fit to the data is in red. This relation is used to adjust the data for these effects of libration.

No averaging of the data was performed to obtain the final adjusted dataset. Included on the following sample of results for FM1 in Figure 14 are the monthly averaged data in blue which aids is verifying that repeatable effects are minimal. Data is plotted as a percentage change of each detector output. Table 6 contains slope changes per decade.

Table 6. Resulting trends in detector stability as extracted from lunar observations

\begin{tabular}{|cccc|}
\hline \multirow{2}{*}{ FM } & \multicolumn{3}{c|}{ Percent change per decade } \\
\cline { 2 - 4 } & TOT & SW & WN \\
\hline $\mathbf{1}$ & 0.192 & 0.353 & 0.518 \\
\hline $\mathbf{2}$ & 0.258 & -0.445 & 0.360 \\
\hline $\mathbf{3}$ & 0.766 & 0.667 & -0.239 \\
\hline $\mathbf{4}$ & 0.473 & N/A & 0.138 \\
\hline
\end{tabular}
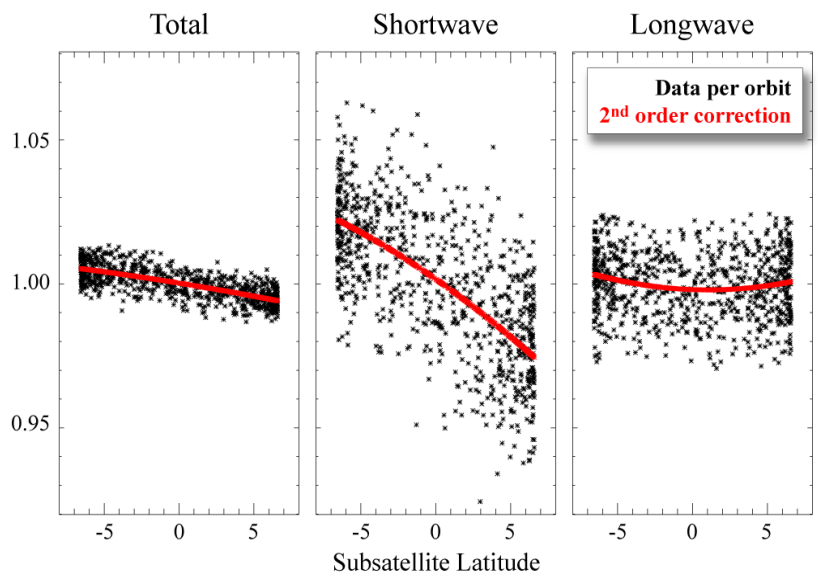

Figure 13. Detector output versus lunar libration latitude
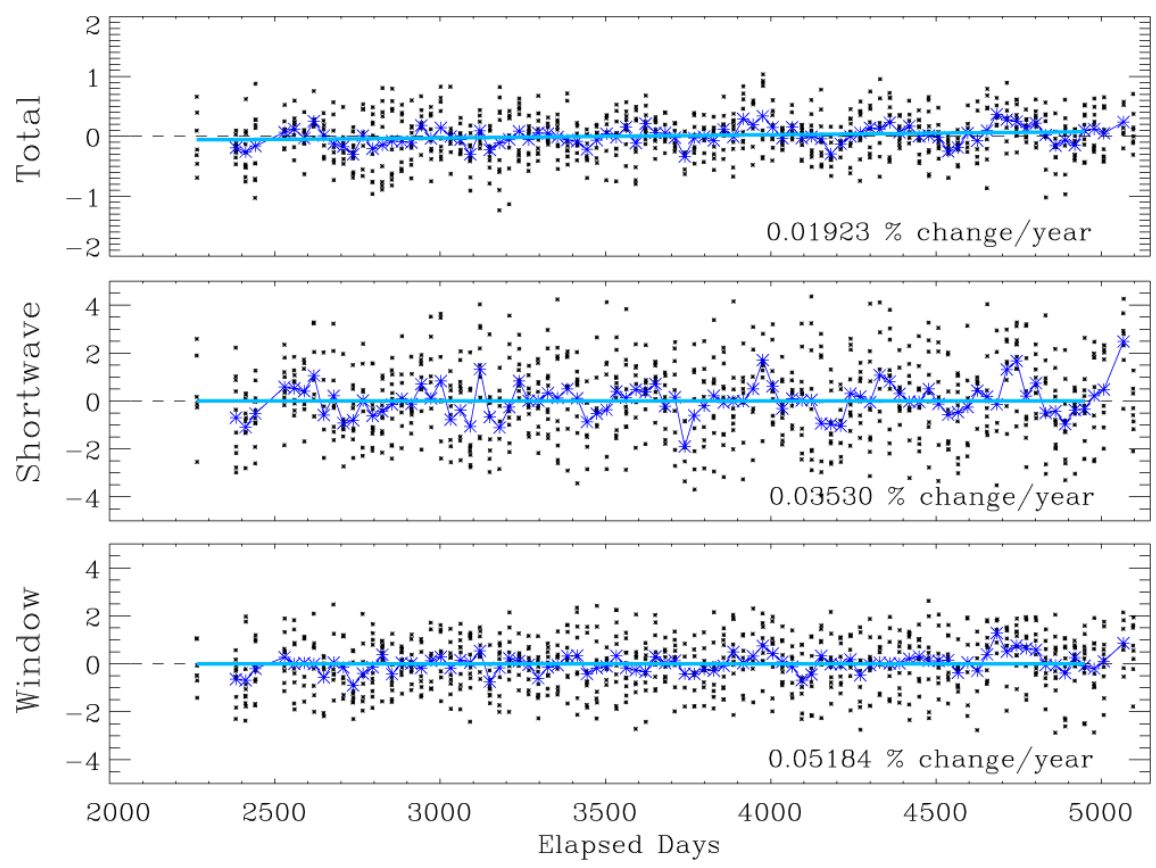

Figure 14. Resulting trends as percent change for FM-1 detector stability extracted from lunar observations

Figure 15 shows the annual running average for each CERES instrument detector. Scale range for the Y-axis has been set to the permitted error ranges allowed in the Radiation Budget Climate Data Record (RBCDR) which are 0.5\% for 
Outgoing Longwave Radiation (OLR) in the total channel detectors and $1.0 \%$ for reflected solar in the shortwave channel detectors. CERES window channels sample a very narrow slice of emitted heat energy and, thus, have no set error criteria as defined in the RBCDR.
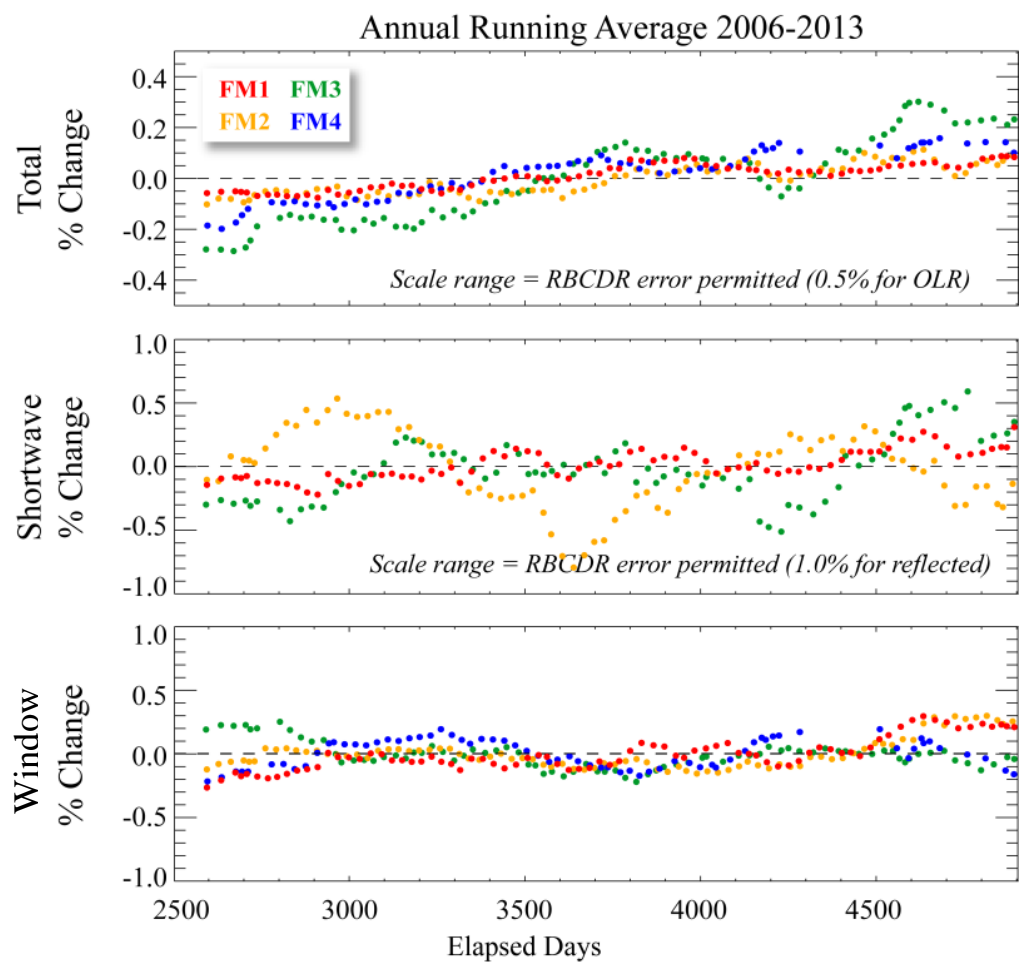

Figure 15. Comparison between CERES detector trending of lunar observations with RBCDR permitted error.

\section{CONCLUSION}

The Moon provides a very versatile, stable source for independent verification of many components of the CERES instruments presently operating on Terra, Aqua and Suomi-NPP spacecraft. The detectors are found to be stable in point response function and pointing accuracy was analyzed using two algorithms with agreed to $0.03^{\circ}$, with the exception of FM-2 and FM-5 window channel detectors which differed by $0.1^{\circ}$.

Alignment of the three channels for each instrument was also validated. Defining this as the difference in azimuth and elevation of the total and shortwave channels and the total and window channels, alignment was found to be within specifications for all instruments. Using the difference in elevation angles to compute geolocation error, the cross-track error is less than $2.5 \mathrm{~km}$ which is smaller than the CERES footprint by an order of magnitude.

Initial variations in lunar observation may appear to be too chaotic to use as a dependable calibration source; however, systematic, mathematical methods are used with lunar orbital data to remove most of these variations. CERES detectors are found to measure lunar irradiance with high precision. These lunar observations serve to validate the consistency of CERES instrument calibrations over time. Linear trends fitted to the final data have slopes of less than $0.8 \%$ per decade. Unlike the telescope alignment and pointing accuracy studies, however, a long-term data set of several years is required to obtain the necessary range of seasonal orbital effects to demonstrate robustness of results for calibration validation.

\section{ACKNOWLEDGEMENTS}

The authors are grateful to the Science Directorate of Langley Research Centre and to the Science Mission Directorate of the Earth Science Division of NASA for the support of the CERES Project. 


\section{REFERENCES}

[1] Loeb N., B. Wielicki, T. Wong and P. Parker, Influence of Gaps in Earth Radiation Budget Climate Data Records. CERES Science Team Meeting, Oct 27, 2008.

[2] Priestley, Kory J., et. al., Radiometric Performance of the CERES Earth Radiation Budget Climate Record Sensors on the EOS Aqua and Terra Spacecraft through April 2007. J.Atmos. Oceanic Technol., 28, 3-21, 2011.

[3] Priestley, K. J., N. G. Loeb, S. Thomas and G. L. Smith, CERES FM5 and FM6: continuity of observations to support a multi-decadal earth radiation budget climate data record, Proc. SPIE 7807, Earth Observing Systems XV, 78070N, Sept. 23, 2010.

[4] Wielicki, B. A., B. R. Barkstrom, E. F. Harrison, R. B. Lee III, G. L. Smith and J. E. Cooper, "Clouds and the Earth's Radiant Energy System (CERES): An Earth Observing System Experiment,” Bull. Amer. Met. Soc., v. 77, 853-868 (1996).

[5] Thomas S., et al., Performance assessment of the Clouds and the Earth's Radiant Energy System (CERES) instruments on Terra and Aqua spacecraft, Proc. SPIE 8866, Earth Observing Systems XVIII, 886606, Sept. 23, 2013

[6] Priestley, K. J., S. Thomas, and G. L. Smith, Validation of Point Spread Functions of CERES Radiometers by the Use of Lunar Observations. Jour. Atmos. \& Ocean. Tech., 27, 1005-1011, 2010.

[7] Daniels, J.L., S. Thomas, G.L. Smith and K.J. Priestley, The point response functions of CERES instruments aboard the Terra and Aqua spacecrafts over the mission-to-date. Imaging Spectrometry XVII, (SPIE Proceedings, Vol. 8515), doi:10.1117/12.928499, Oct. 2012

[8] Daniels, J.L., G.L. Smith, S. Thomas, and K.J. Priestley, Using Lunar Observations to Validate CERES Pointing Accuracy. Submitted to Trans. on Geos. and Remote Sensing, 2014.

[9] Smith, G. L. , G. L. Peterson, R. B. Lee and B. R. Barkstrom, “Optical design of the CERES telescope”, Proc. SPIE, 4483-32 (2001).

[10] Daniels, J.L., G.L. Smith, S. Thomas, and K.J. Priestley, Using Lunar Observations to Validate In-flight Calibrations of Clouds and the Earth's Radiant Energy System Instruments. Submitted to Trans. on Geos. and Remote Sensing, 2014.

[11] Kieffer, H. H., Photometric Stability of the Lunar Surface. Icarus (130: 323-327), 1997 